\title{
Deposition of elements for a thermoelectric generator via laser-induced forward transfer
}

\author{
M. Feinaeugle ${ }^{1}$, C. L. Sones ${ }^{1}$, E. Koukharenko ${ }^{2}$, B. Gholipour ${ }^{1}$, D. W. Hewak ${ }^{1}$, R. W. Eason ${ }^{1}$ \\ ${ }^{1}$ Optoelectronics Research Centre, University of Southampton, Southampton, SO17 IBJ, UK \\ ${ }^{2}$ School of Electronics and Computer Science, University of Southampton, Southampton, SO17 1BJ, UK \\ Author (M. Feinaeugle) e-mail address: mf2v09@orc.soton.ac.uk, Tel: (+44) 0238059 9091, Fax: (+44) 02380593142
}

\begin{abstract}
Films of thermoelectric bismuth selenide and bismuth telluride have been transferred by laser-induced forward transfer onto glass and polymer substrates. Influence of transfer on thermoelectric properties and morphology of the material was compared.

OCIS codes: (350.3390) Laser materials processing; (310.6845) Thin film devices and applications
\end{abstract}

\section{Introduction}

Laser-induced forward transfer (LIFT) is a technique that has been used in the prototyping and repair of thin film electrical and optical devices [1]. The technique is an additive direct-write method for micro-fabrication without using vacuum technology and any lithographic or annealing process steps. LIFT offers the possibility of intact printing of a portion of a substrate (referred to as the donor) onto an acceptor substrate (the receiver). Thus, it may enable the transfer of donor layers that have been previously grown with a pre-defined phase, pixel structure or specific properties as the LIFT-transferred deposit may only be affected within a thin surface region when absorbing the laser pulse at the interface between the donor and the transparent donor substrate (the carrier) [2]. LIFT could thus enable the fabrication of entire electronic or optical devices without further post-deposition processing steps.

In order to demonstrate the feasibility of LIFT in the fabrication of a thermoelectric energy harvesting device [3] we have shown, in the following, LIFT of the thermoelectric chalcogenide donors bismuth selenide $\left(\mathrm{Bi}_{2} \mathrm{Se}_{3}\right)$ and bismuth telluride $\left(\mathrm{Bi}_{2} \mathrm{Te}_{3}\right)$ and have compared the Seebeck coefficients of the original donor layer and the resultant transferred pixel. Furthermore we have studied the morphology of the donor and we report our progress towards LIFT fabrication of a thermoelectric generator that can be used for energy harvesting.

\section{Methods}

The setup for our LIFT experiments consisted of a pulsed $\mathrm{KrF}$ excimer laser source with a wavelength of $248 \mathrm{~nm}$ and $20 \mathrm{~ns}$ pulse duration. The maximum pulse energy used was $400 \mathrm{~mJ}$ with a quasi-homogeneous beam area of $2.4 \mathrm{~cm}^{2}$. This pulse was imaged via an aperture mask onto the carrier/donor interface via a plano-convex lens. The energy of the pulse was controlled by introducing attenuators of fused silica into the beam path. The position of the deposits and the focus of the beam were manipulated by adjusting a three-axis stage.

Donor films with a thickness of $\sim 0.4-1.4 \mu \mathrm{m}$ were previously prepared by sputtering layers of $\mathrm{Bi}_{2} \mathrm{Se}_{3}$ and $\mathrm{Bi}_{2} \mathrm{Te}_{3}$ onto UV-transparent fused silica substrates. Various receivers were used including glass, silicon and Parafilm-coated (Pechiney Plastic Packaging) glass substrates which were placed in contact or at a distance of some microns from the donor surface.

The experiments were conducted with a laser fluence in the range of $50-300 \mathrm{~mJ} / \mathrm{cm}^{2}$. Following successful LIFT transfer, the deposits and the donor films were imaged via optical and scanning electron microscopy. The film composition was determined using energy-dispersive X-ray spectroscopy (EDX) and the crystal structure of the films was measured by X-ray diffraction (XRD). We measured the Seebeck coefficient with a bespoke setup in which the voltage over a thin film was measured for a temperature difference applied between opposite sides of a sample.

\section{Results and Discussion}

Thin donor layers $(0.4 \mu \mathrm{m})$ of $\mathrm{Bi}_{2} \mathrm{Te}_{3}$ were transferred onto glass as a receiver with a donor-receiver separation of $\sim 1 \mu \mathrm{m}$. We identified a threshold of $\sim 60 \mathrm{~mJ} / \mathrm{cm}^{2}$ for material release with pixels transferred with only partial melting. However, the material transferred did not adhere well onto the receiver. For a fluence of $100-300 \mathrm{~mJ} / \mathrm{cm}^{2}$ the laser energy density was high enough to completely melt and fragment the donor which resulted in its incomplete release from the carrier and also undesirable re-deposition as material was found on both the receiver and the donor in a resolidified state. However, adhesion of the molten layers on the glass receiver was good.

For a layer of $\sim 1.4 \mu \mathrm{m}$ thick $\mathrm{Bi}_{2} \mathrm{Se}_{3}$ or $\mathrm{Bi}_{2} \mathrm{Te}_{3}$ the threshold fluence for material ejection, for which the donor was transferred in an intact state, was $\sim 70 \mathrm{~mJ} / \mathrm{cm}^{2}$. The receiver, a Parafilm polymer-coated glass slide was kept in close contact with the donor. Over the entire fluence range of $\left(70-300 \mathrm{~mJ} / \mathrm{cm}^{2}\right)$, we observed that the material was 
released from the donor. However for higher fluences, the surface of the transferred donor experienced stronger melting. Furthermore the pixel underwent strong fragmentation and adhesion onto the receiver decreased at the same time. The LIFTed deposits had an area of up to $\sim 12 \mathrm{~mm}^{2}$.

The measured Seebeck coefficients of the donor films showed a value of $-121 \mu \mathrm{V} / \mathrm{K}$ and $-64 \mu \mathrm{V} / \mathrm{K}$ for $\mathrm{Bi}_{2} \mathrm{Se}_{3}$ and $\mathrm{Bi}_{2} \mathrm{Te}_{3}$ respectively. These values when measured for the LIFTed pixels decreased to $-93 \mu \mathrm{V} / \mathrm{K}$ and $-49 \mu \mathrm{V} / \mathrm{K}$ respectively, which is about $80 \%$ of the initial value of the donor. The negative sign in the coefficient indicates that the films are n-type.

The polycrystalline structure of the donor films was confirmed via SEM imaging. Through the absorption of the laser pulse during transfer, a fraction of the pixel had been melted as seen in Fig 1a). An image of $\mathrm{LIFTed}_{\mathrm{Bi}} \mathrm{Te}_{3}$ deposits on a polymer-coated glass receiver transferred in a fluence range of $60-300 \mathrm{~mJ} / \mathrm{cm}^{2}$ is shown in Fig. $1 \mathrm{~b}$ ).
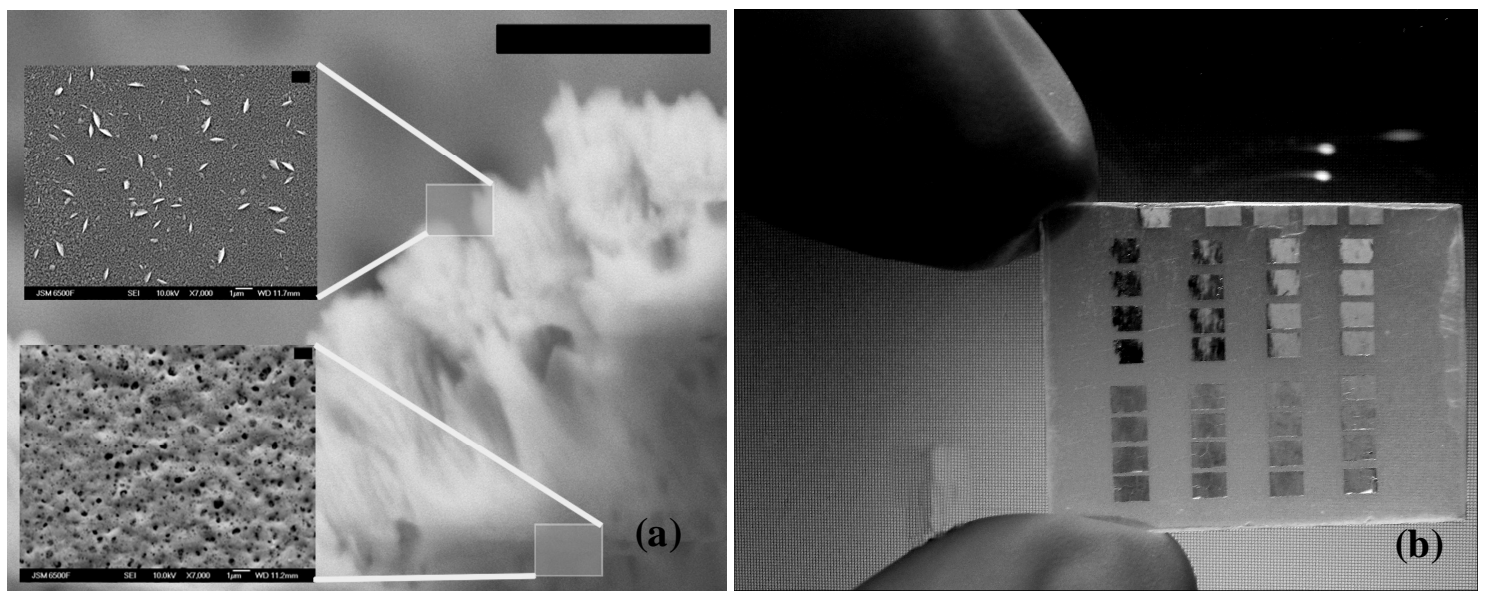

Fig. 1. (a): SEM image of the edge of a $\mathrm{Bi}_{2} \mathrm{Te}_{3}$ pixel, tilted at an angle of $\sim 45$ degrees and previously transferred at a fluence of $\sim 80$ $\mathrm{mJ} / \mathrm{cm}^{2}$. The two insets are images of lower magnification of two selected surface regions of the pixel. The surface that was previously facing the carrier (bottom inset) experienced melting due to the absorption of the ns-pulse. The edge and the opposite side show the initial polycrystalline

structure of the chalcogenide donor film (e.g. top inset). All three scale bars are $1 \mu \mathrm{m}$ wide. (b): Image of a polymer-coated (Parafilm) glass receiver with $1 \mu \mathrm{m}$ thick $\mathrm{Bi}_{2} \mathrm{Te}_{3}$ pixels transferred via ns-LIFT in a fluence range of $60-300 \mathrm{~mJ} / \mathrm{cm}^{2}$. The dimension of a single transferred pixel is $2 \mathrm{~mm} \times 1.5 \mathrm{~mm}$.

The XRD analysis performed to evaluate films microstructure showed a diffraction peak at $2 \theta=27$ degrees for both the donor films and the transferred $\mathrm{Bi}_{2} \mathrm{Te}_{3}$ deposits. This peak relates to the rhombohedral structure of $\mathrm{Bi}_{2} \mathrm{Te}_{3}$ crystals with the R3-m space group [4].

Our experiments show that chalcogenide donors can be successfully LIFT transferred without major degradation of their physical or thermoelectric properties, and this is sufficient for the use of such LIFTed pixels as elements of a thermoelectric generator. Future work will concentrate on the fabrication of an entire thermoelectric generator including thermoelectric p-type and n-type elements and metal electrodes within the same device.

\section{Summary}

We have demonstrated successful LIFT printing of $\mathrm{Bi}_{2} \mathrm{Se}_{3}$ and $\mathrm{Bi}_{2} \mathrm{Te}_{3}$ thermoelectric materials with an area of up to $\sim 12 \mathrm{~mm}^{2}$ onto glass and polymer substrates. Intact and molten transfer regimes were identified and the influence of transfer parameters, receiver choice and the specific material properties have been demonstrated. We show that the Seebeck coefficient of the transferred chalcogenide donor pixels was up to a value of $80 \%$ of the initial value when compared to the donor.

\section{References}

[1] C. B. Arnold, P. Serra and A. Pique, "Laser direct-write techniques for printing of complex materials," MRS Bull. 32, 23-31 (2007).

[2] K. S. Kaur, M. Feinaeugle, D.P. Banks, J.Y. Ou, F. Di Pietrantonio, E. Verona, C.L. Sones and R.W. Eason, "Laser-induced forward transfer of focussed ion beam pre-machined donors," Appl Surf Sci 257, 6650-6653, (2011).

[3] G. J. Snyder and E.S. Toberer, “Complex thermoelectric materials,” Nat. Mater. 7, 105-114, (2008).

[4] P. W. Lange, Ein Vergleich zwischen $\mathrm{Bi}_{2} \mathrm{Te}_{3}$ und $\mathrm{Bi}_{2} \mathrm{Te}_{2} \mathrm{~S}$,“ Naturwissenschaften 27, 133-134 (1939). 